\title{
Oxygen flux in the solar wind: Ulysses observations
}

\author{
Rudolf von Steiger, ${ }^{1}$ Thomas H. Zurbuchen, ${ }^{2}$ and David J. McComas ${ }^{3,4}$ \\ Received 3 September 2010; revised 28 September 2010; accepted 1 October 2010; published 17 November 2010.
}

[1] We use the complete set of Ulysses solar wind data to conduct a comprehensive determination of the oxygen flux in relation to the proton flux in the solar wind during a wide range of solar activity levels. The data cover the heliosphere between 1.3 and 5.4 $\mathrm{AU}$ and, due to the unique orbit of Ulysses, all of the heliographic latitudes within $\pm 80^{\circ}$. We find log-normal distributions for $\mathrm{O}$ and $\mathrm{H}$ daily flux values, but with significant differences between slow and fast wind. Coronal hole-associated fast wind has a distribution that is approximately three times narrower than the one of slow wind associated with streamers. Finally, we derive the solar oxygen abundance, finding a value of $8.82 \pm$ 0.08 , and discuss this value in comparison with the results of spectroscopic determinations. Citation: von Steiger, R., T. H. Zurbuchen, and D. J. McComas (2010), Oxygen flux in the solar wind: Ulysses observations, Geophys. Res. Lett., 37, L22101, doi:10.1029/2010GL045389.

\section{Introduction}

[2] Oxygen is the third most abundant element or, astronomically speaking, the most abundant "metal" in the Sun. Only helium and hydrogen are more abundant, by roughly a factor 100 and 1000 , respectively. Yet the solar oxygen abundance, or equivalently the $\mathrm{H} / \mathrm{O}$ ratio, is relatively uncertain for reasons discussed later. This is unfortunate since the $\mathrm{H} / \mathrm{O}$ value is a key ingredient and constraint to several types of studies such as the interpretation of helioseismological analyses, our understanding of galactic chemical evolution, or also of the early history of the solar system.

[3] There are generally two methods for measuring the solar H/O ratio: Spectroscopy of the solar atmosphere and in-situ observations of the solar wind. The former method applied to the photosphere has yielded an oxygen abundance from 8.93 [Anders and Grevesse, 1989], 8.83 [Grevesse and Sauval, 1998], 8.66 [Grevesse et al., 2007], to 8.69 [Asplund et al., 2009] over the last two decades, i.e., a decrease by more than $40 \%$. Here, the abundance of an element $\mathrm{X}$ is defined as $12-\log [\mathrm{H} / \mathrm{X}]$. Typically, the spectroscopic determinations of abundances cannot be obtained from a simple comparison of the strength of spectral absorption lines, but depend strongly on accurate modeling of the

\footnotetext{
${ }^{1}$ International Space Science Institute, Bern, Switzerland.

${ }^{2}$ Department of Atmospheric, Oceanic and Space Sciences, University of Michigan, Ann Arbor, Michigan, USA.

${ }^{3}$ Space Science and Engineering Division, Southwest Research Institute, San Antonio, Texas, USA.

${ }^{4}$ Department of Physics and Astronomy, University of Texas at San Antonio, San Antonio, Texas, USA.

Copyright 2010 by the American Geophysical Union. 0094-8276/10/2010GL045389
}

optical emission from and through the solar atmosphere. The newer, lower values are based on computations from an improved, 3d, and non-LTE model of the solar atmosphere. Spectroscopic abundances can also be determined in the outer solar corona. Antonucci et al. [2006] find an oxygen abundance of 8.8 from observations with SoHO UVCS.

[4] The second methodology makes use of the fact that the solar wind carries a sample of solar material into interplanetary space, where it is accessible to in-situ analysis using space-borne mass spectrometers. There are two difficulties with this approach: first, it must be assumed that the solar wind is a genuine, unfractionated sample of solar material, or an appropriate correction for fractionation needs to be made. Second, hydrogen and oxygen ions are usually measured by different types of sensors due to their very large abundance differences: a fast plasma instrument and a slower composition sensor with higher sensitivity and the ability to resolve heavy ions, thus raising issues about the intercalibration of these instruments. For this reason there are few values in the literature: Bame et al. [1975] gave H/O = 1900, corresponding to an oxygen abundance of 8.72 , obtained with a single sensor in the predominantly slow solar wind with a low kinetic temperature near the ecliptic plane. A more sophisticated measurement technique is needed for a reliable determination of this value.

[5] In this paper we report on the first comprehensive determination of the flux of protons and of oxygen ions in the solar wind and their ratio using the SWOOPS [Bame et al., 1992] and the SWICS [Gloeckler et al., 1992] instruments on the Ulysses mission [Wenzel et al., 1992]. Ulysses was launched in 1990 and, after a Jupiter fly-by in 1992 , orbited the Sun on a unique orbit that is inclined by $80^{\circ}$ to the solar equator at a distance of between 1.34 and 5.41 AU. Until the end of the mission on June 30, 2009, it had flown almost three polar orbits around the Sun. The first and third set of polar passes occurred near solar minimum conditions and gave rise to similar observations with small but interesting differences, while the second one coincided with the maximum of solar cycle 23 and gave a remarkably different picture of the heliosphere [McComas et al., 2001, 2008; von Steiger and Zurbuchen, 2010]. The results obtained from Ulysses therefore apply to all phases of the solar cycle and to all latitudes in the heliosphere, which makes them ideal for long-term studies such as this one.

\section{H and $O$ Fluxes in the Solar Wind}

[6] In Figure 1 we present a survey of the physical quantities used in this work. Figure 1a shows the solar wind speed as measured by SWOOPS, and Figure $1 \mathrm{~b}$ shows the freeze-in temperature as inferred from the $\mathrm{O}^{7+} / \mathrm{O}^{6+}$ measured by SWICS using the ionization equilibrium tables of Mazzotta et al. [1998]. Together Figures 1a and 1b illustrate that the solar wind is basically a two-state phenomenon: 


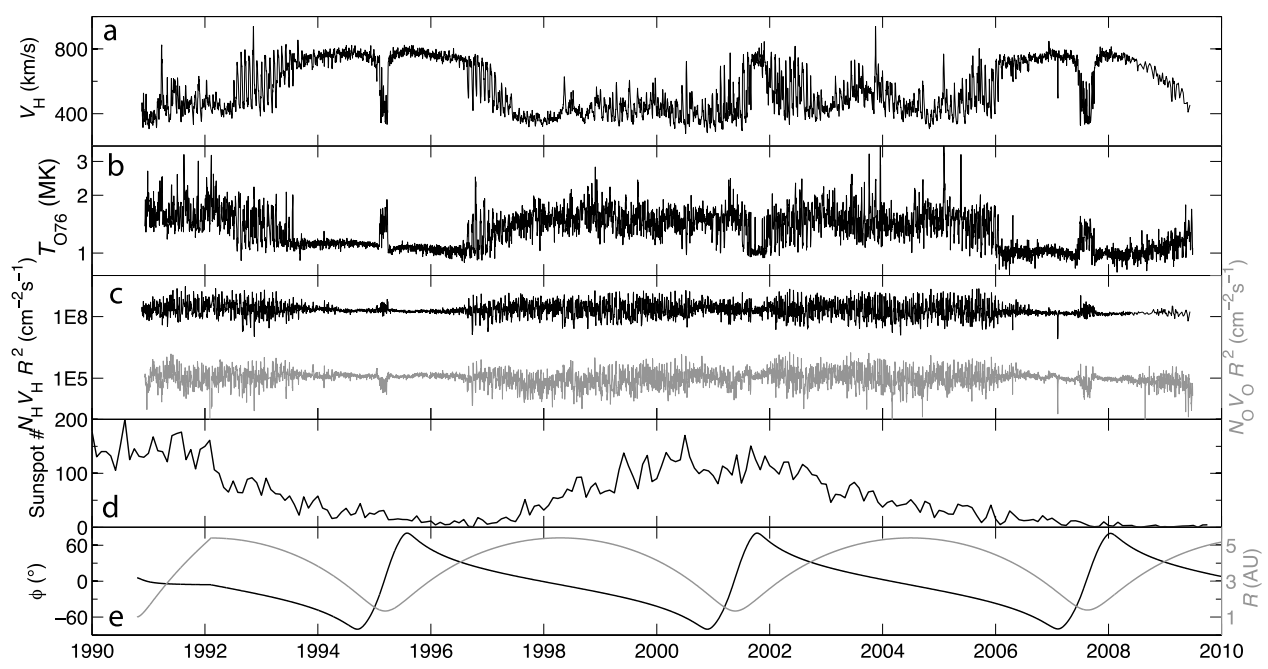

Figure 1. Overview of solar wind parameters during the entire Ulysses mission. (a) Solar wind speed, (b) freezing-in temperature as inferred from the $\mathrm{O}^{7+} / \mathrm{O}^{6+}$ charge state ratio, (c) fluxes of protons (black) and of oxygen ions (grey), both scaled to $1 \mathrm{AU}$, (d) mean monthly sunspot number, and (e) Ulysses heliographic latitude (black) and heliocentric distance (grey).

fast, steady wind from the relatively cool coronal holes, and slow, but more variable wind from the coronal streamer belt region and near solar maximum when polar coronal holes are not a dominant source of solar wind [Zurbuchen et al., 2002; Zurbuchen, 2007]. Figure 1c shows the flux of solar wind protons measured by SWOOPS (above) and of oxygen ions measured by SWICS (below), both scaled to 1 AU by multiplication with the squared heliocentric distance of Ulysses. From Figure 1c the near-constancy of the (scaled) solar wind flux, already noted by Schwenn [1990], becomes immediately apparent. Another striking feature is the fact that there is much less flux variability in the fast wind as compared to the slow wind. Figure 1d gives the solar activity using the mean monthly sunspot number from http:// www.swpc.noaa.gov/SolarCycle/, and Figure 1e gives the Ulysses heliographic latitude and distance. Together they show the fortuitous matching between Ulysses' polar passes and the sunspot activity already mentioned.

[7] Based on these data, we have constructed histograms of the daily values of the proton flux and of the flux of oxygen ions. For the former we have used the hourly values of the proton density, $N_{\mathrm{H}}$, proton speed, $V_{\mathrm{H}}$, and heliocentric distance, $R$, given in the Ulysses public archive (http:// helios.estec.esa.nl/ulysses/archive/) and properly averaged to obtain daily values, and for the latter we have summed over daily values of the fluxes of $\mathrm{O}^{6+},{ }^{7+}$, and ${ }^{8+}$. The reason for averaging the SWOOPS hourly data to daily values is the desire to use the same resolution for both time series at a value for which $\mathrm{O}$ fluxes are reliably determined. The histograms of daily values are then separated according to solar wind type and shown in Figure 2.

[8] The separation into the two solar wind types was done as follows: Even though the two types are usually referred to as "fast" and "slow", it has been shown earlier that a separation based on the coronal temperature is superior [Geiss et al., 1995; Zurbuchen et al., 2002]. We have recently refined the separation criterion, finding that the following product of two charge state ratios yields a very successful
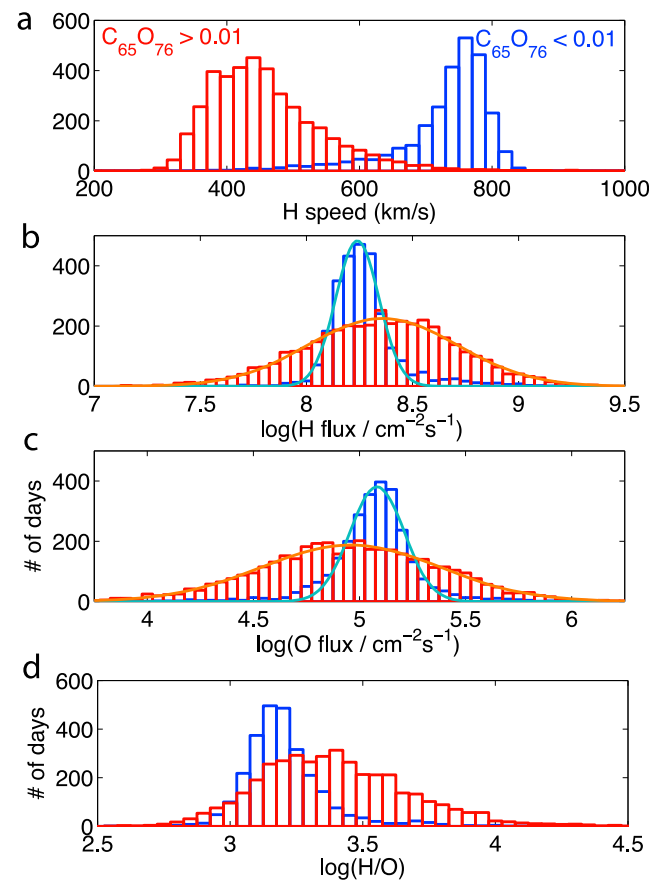

Figure 2. Distributions of daily averages of (a) the solar wind speed, (b) the proton flux, and (c) the flux of oxygen ions, obtained in fast (blue) and slow (red) solar wind as defined by equation (1). In both wind types, and for both elements, the flux distributions are log-normals to a high degree of accuracy as indicated by the cyan and orange fitting curves, respectively, but the slow wind distributions are much wider than the ones in the fast wind. (d) The distributions of daily values of the $\mathrm{H} / \mathrm{O}$ flux ratios; the average $\mathrm{H} / \mathrm{O}$ value differs in the two solar wind types, indicating that at least one of them must be fractionated relative to the solar photosphere. 
Table 1. Parameters of the Lognormal Distribution Functions Given in Figure 2 for Protons and Oxygen Ions in Both the Fast and the Slow Solar Wind as Defined by Equation (1), and the Solar Wind H/O Ratio Obtained From the Average of the Daily Flux Ratios Also Shown in Figure 2

\begin{tabular}{lcccc}
\hline Element & Mean "fast" & Width "fast" & Mean "slow" & Width "slow" \\
\hline$\langle\log \mathrm{H}\rangle$ & 8.240 & 0.102 & 8.360 & 0.353 \\
$\langle\log \mathrm{O}\rangle$ & 5.083 & 0.128 & 4.960 & $\begin{array}{l}0.422 \\
+1900 \\
\langle\mathrm{H} / \mathrm{O}\rangle\end{array}$ \\
\hline
\end{tabular}

separation and is less prone to statistical effects than other methods:

$$
\frac{\mathrm{O}^{7+}}{\mathrm{O}^{6+}} \cdot \frac{\mathrm{C}^{6+}}{\mathrm{C}^{5+}} \begin{cases}<0.01 & \rightarrow \text { fast wind } \\ >0.01 & \rightarrow \text { slow wind }\end{cases}
$$

[von Steiger, 2008]. In this work we exclusively use this criterion; the solar wind speed, which might lead to a criterion with radial dependence due to stream-stream interactions and other dynamic effects, is merely shown for illustrative purposes.

[9] In Figure 2a we show histograms of the solar wind speed, with the red histogram representing the slow wind with hotter freeze-in temperatures and the blue histogram representing the fast wind originating from the cool coronal hole areas, as defined in equation (1). There are 2677 daily samples of fast wind and 3973 samples of slow wind in our data set, which means that we have less than $2 \%$ of data gaps in this analysis. Evidently the two histograms have very little overlap, which means that a separation by solar wind speed with a threshold at $630 \mathrm{~km} / \mathrm{s}$, say, would have worked almost as well for this particular data set. However, this is primarily due to the large proportion of data from high latitudes and at low solar activity in the Ulysses dataset. When analyzing data from a mission at $1 \mathrm{AU}$ such as ACE there might be much more overlap between the two histograms due to fast, hot CMEs that might be falsely identified as coronal hole-associated based on speed alone.

[10] Figure 2b shows histograms of the scaled logarithmic proton flux for the two solar wind types. In both cases we find that the distribution of daily samples can be fitted very accurately with a Gaussian, i.e., the flux distributions are log-normal. The fitting curves are also indicated in Figure 2 and their parameters are given in Table 1 . The quality of the fits is very good, with the linear correlation coefficient between the model distribution and the data $>0.99$ in both cases. But the most interesting result is the observation that the widths of the two log-normal distribution functions are vastly different, with the slow wind distribution more than three times wider than the fast wind distribution. In other words, $67 \%$ of the fast wind samples are within a factor of 1.26 of the mean, but $67 \%$ of slow wind samples scatter by the much larger factor of 2.25 around their mean. An analysis of proton flux data with one-hour resolution was found to yield identical results to within the error bars of the fit. Thus, the log-normal character of these distributions, and particularly the significant differences between slow and fast wind, are intrinsic properties of solar wind.

[11] Log-normal distributions of solar wind parameters have been discussed previously. Burlaga and Lazarus [2000] found that the solar wind density and speed as measured on the Voyager mission follow log-normal dis- tributions. They also noted that the distributions become bimodal when a significant amount of fast wind is part of the sample. They did not give a theoretical explanation of the occurrence of log-normals but Burlaga [2001] noted that a binomial cascade model would produce such distributions. Here, we add very important constraints to this discussion. The underlying processes leading to these distributions, e.g., by an iterative redistribution of mass flux, must be evolved close to equilibrium as indicated by the high accuracy of the lognormals, but the parameters characterizing these processes must be significantly different in the fast and slow wind.

[12] In Figure 2c we show the same histograms for the logarithm of the scaled oxygen fluxes. Again we find lognormal distributions of equally high quality as for protons, and again the slow wind distributions is more than three times wider than the fast wind distribution. Both distributions are somewhat wider than the corresponding proton distributions, but more importantly their centers are shifted relative to each other. While the average proton flux in the slow wind is higher by some $32 \%$ than in the fast wind this relation is reversed in the case of $\mathrm{O}$. Thus, the $\mathrm{H} / \mathrm{O}$ ratio is observed to be differently fractionated relative to the Sun in the two solar wind types.

\section{Solar Wind Oxygen Abundance}

[13] We now find the oxygen abundance (or $\mathrm{H} / \mathrm{O}$ ratio) by dividing each daily proton flux (i.e., the arithmetic mean of the 24 hourly values) by the corresponding oxygen flux and find the average of these ratios. In Figure $2 \mathrm{~d}$ we show the distributions of the daily values of $\mathrm{H} / \mathrm{O}$. The two distributions for fast and slow wind are not quite as log-normal as compared to the individual fluxes, but they are significantly narrower than the individual flux distributions, notably in the slow wind case; their parameters are also given in Table 1. The widths of the distributions mainly represent the natural variability of the daily values of the $\mathrm{H} / \mathrm{O}$ ratio, and some potential measurement errors discussed later.

[14] We find that our slow wind value of $\mathrm{H} / \mathrm{O}$ agrees with Bame et al.'s [1975] value to within the error bars. This might be expected because that value was also obtained during selected periods of quiet, slow solar wind; only at such times the kinetic temperature of the wind was sufficiently low as to allow the identification of heavy ions such as $\mathrm{O}^{6+}$. To this we may add a contribution for $\mathrm{O}^{7+}$, which could not be observed with Bame's instrument due to its proximity to the much higher peak of $\mathrm{He}^{++}$. The $\mathrm{O}^{7+} / \mathrm{O}^{6+}$ ratio is typically $20 \%$ in the slow wind, and when adding this to the Bame value it becomes almost identical to our slow wind value.

[15] However, we expect the fast wind $\mathrm{H} / \mathrm{O}$ value to be a much closer representation of the solar $\mathrm{H} / \mathrm{O}$ value for two reasons: First, it is now a well-established fact that abundances of heavy ions in the fast solar wind are less affected by chromospheric fractionation such as the observed first ionization potential (FIP) effect [von Steiger et al., 2000]. Second, the ionization potentials of $\mathrm{H}$ and $\mathrm{O}$ are nearly identical so no fractionation based on UV ionization should be expected, which is often at the heart of FIP fractionation models [e.g., von Steiger and Geiss, 1989]. Although the possibility remains that the fast wind value is also frac- 
tionated, albeit to a lesser extent than the slow wind value, we therefore identify our fast wind value with the solar $\mathrm{H} / \mathrm{O}$ ratio. The statistical uncertainty of our value is negligible considering the large amount of data, but of course there remains a systematic uncertainty. For SWICS this was determined recently through a detailed analysis by von Steiger and Zurbuchen [2010] at 15\% for the absolute flux of oxygen, and assuming a conservative $\sim 10 \%$ for the SWOOPS proton flux we obtain

$$
\mathrm{H} / \mathrm{O}=1500 \pm 300 .
$$

Our value of the $\mathrm{H} / \mathrm{O}$ ratio corresponds to a solar oxygen abundance of $8.82 \pm 0.08$, which is just barely in agreement with the error bars of the newest spectroscopic value of $8.69 \pm 0.05$ by Asplund et al. [2009]. Note that any residual fractionation in (2) would increase that discrepancy because the actual solar $\mathrm{H} / \mathrm{O}$ value would then be lower, or the solar oxygen abundance higher. On the other hand our value is very much consistent with the previous determination of Grevesse and Sauval [1998]. Recent observations with UVCS on the SoHO mission tend to support our value by also favoring a higher oxygen abundance [Antonucci, 2007].

\section{Discussion and Conclusions}

[16] The solar oxygen abundance is an important parameter for several types of studies, but it is unfortunately still not known with a very high accuracy. Oxygen makes the biggest contribution to the solar metallicity and is therefore important to models of galactic chemical evolution. Further, the protosolar abundance of oxygen (and other heavy elements) is fundamental for models of the origin and evolution of the solar system as it represents the baseline from which all planetary bodies were formed [Geiss and Gloeckler, 2007]. Third, oxygen is a source of opacity in the solar interior and thus important for helioseismology [Gough, 1998].

[17] The downward revision of the oxygen abundance (and, to a lesser extent, of other metals) first proposed by Asplund et al. [2004] implies a lower metallicity of the Sun, $Z=0.013$, compared to $Z=0.020$ from Anders and Grevesse's [1989] abundances. This has the advantage of bringing the Sun into closer agreement with OB stars in the solar neighborhood [Asplund et al., 2009]. On the other hand the lower oxygen abundance also leads to a significant discrepancy between standard solar models and helioseismology results that needs to be addressed [Bahcall et al., 2005]. In order to reconcile this discrepancy these authors proposed an increase of the solar neon abundance by $0.4-0.5 \mathrm{dex}$, or a factor of $\sim 3$. However, solar wind observations of the $\mathrm{Ne} / \mathrm{O}$ ratio [von Steiger et al., 2000] argue against such a large neon abundance correction. Note that the solar $\mathrm{Ne}$ abundance is even less well known than that of $\mathrm{O}$ because the element does not have any absorption lines at photospheric temperatures.

[18] In this paper we have presented the first comprehensive determination of the solar wind $\mathrm{H} / \mathrm{O}$ ratio using the long-term, stable, and continuous dataset obtained with the Ulysses mission. We find that the fluxes of these two elements have log-normal distributions to a very high degree, broad in the slow wind and narrow in the fast wind. This is a highly important result in the context of the structure of the solar wind source region and its dynamic evolution in the inner heliosphere, which will be first explored by the Solar Probe+ [McComas et al., 2007] and the Solar Orbiter missions. The main result is a new determination of the solar oxygen abundance of $8.82 \pm 0.08$, which is higher than the latest spectroscopic value [Asplund et al., 2009] by more than 0.1 dex. Our $\mathrm{O}$ abundance is not at odds with helioseismology results and thus removes the need for an ad-hoc increase of the solar $\mathrm{Ne}$ abundance postulated by Bahcall et al. [2005].

[19] Acknowledgments. This work is supported, in part, by JPL contract 1268016 and NASA grant NNX09AH72G. THZ acknowledges the hospitality of the International Space Science Institute in Bern, Switzerland, where most of his contribution to this work was performed. We acknowledge the many individuals who, during more than 30 years, have contributed a truly unique data set from Ulysses and particularly the SWICS and the SWOOPS instruments.

\section{References}

Anders, E., and N. Grevesse (1989), Abundances of the elements: Meteoritic and solar, Geochim. Cosmochim. Acta, 53, 197-214, doi:10.1016/ 0016-7037(89)90286-X.

Antonucci, E. (2007), Wind in the solar corona: Dynamics and composition, Space Sci. Rev., 124, 35-50, doi:10.1007/s11214-006-9098-6.

Antonucci, E., L. Abbo, and D. Telloni (2006), Oxygen abundance and energy deposition in the slow coronal wind, Astrophys. J., 643, 1239-1244, doi:10.1086/503186.

Asplund, M., N. Grevesse, A. J. Sauval, C. Allende Prieto, and D. Kiselman (2004), Line formation in solar granulation. IV. [O I], O I and $\mathrm{OH}$ lines and the photospheric O abundance, Astron. Astrophys., 417, 751-768, doi:10.1051/0004-6361:20034328.

Asplund, M., N. Grevesse, A. J. Sauval, and P. Scott (2009), The chemical composition of the Sun, Annu. Rev. Astron. Astrophys., 47, 481-522, doi:10.1146/annurev.astro.46.060407.145222.

Bahcall, J. N., S. Basu, and A. M. Serenelli (2005), What is the neon abundance of the Sun?, Astrophys. J., 631, 1281, doi:10.1086/431926.

Bame, S. J., J. R. Asbridge, W. C. Feldman, M. D. Montgomery, and P. D. Kearney (1975), Solar wind heavy ion abundances, Sol. Phys. 43, 463-473, doi:10.1007/BF00152368.

Bame, S. J., D. J. McComas, B. L. Barraclough, J. L. Phillips, K. J. Sofaly, J. C. Chavez, B. E. Goldstein, and R. K. Sakurai (1992), The Ulysses solar wind plasma experiment, Astron. Astrophys. Suppl. Ser., 92, 237-265.

Burlaga, L. (2001), Lognormal and multifractal distributions of the heliospheric magnetic field, J. Geophys. Res., 106, 15,917-15,927, doi:10.1029/2000JA000107.

Burlaga, L. F., and A. J. Lazarus (2000), Lognormal distributions and spectra of solar wind plasma fluctuations: Wind 1995-1998, J. Geophys Res., 105, 2357-2364, doi:10.1029/1999JA900442.

Geiss, J., and G. Gloeckler (2007), Linking primordial to solar and galactic composition, Space Sci. Rev., 130, 5-26, doi:10.1007/ s11214-007-9235-x.

Geiss, J., G. Gloeckler, and R. von Steiger (1995), Origin of the solar wind from composition data, Space Sci. Rev., 72, 49-60, doi:10.1007/ BF00768753.

Gloeckler, G., et al. (1992), The solar wind ion composition spectrometer, Astron. Astrophys. Suppl. Ser., 92, 267-289.

Gough, D. (1998), On the composition of the solar interior, Space Sci. Rev., 85, 141-158, doi:10.1023/A:1005105224272.

Grevesse, N., and A. J. Sauval (1998), Standard solar composition, Space Sci. Rev., 85, 161-174, doi:10.1023/A:1005161325181.

Grevesse, N., M. Asplund, and A. J. Sauval (2007), The solar chemical composition, Space Sci. Rev., 130, 105-114, doi:10.1007/s11214-007-9173-7.

Mazzotta, P., G. Mazzitelli, S. Colafrancesco, and N. Vittorio (1998), Ionization balance for optically thin plasmas: Rate coefficients for all atoms and ions of the elements $\mathrm{H}$ to Ni, Astron. Astrophys. Suppl., 133, 403-409.

McComas, D. J., R. Goldstein, J. T. Gosling, and R. M. Skoug (2001), Ulysses' second orbit: Remarkably different solar wind, Space Sci. Rev., 97, 99-103, doi:10.1023/A:1011826111330.

McComas, D. J., et al. (2007), Understanding coronal heating and solar wind acceleration: Case for in situ near-Sun measurements, Rev. Geophys., 45, RG1004, doi:10.1029/2006RG000195. 
McComas, D. J., R. W. Ebert, H. A. Elliott, B. E. Goldstein, J. T. Gosling, N. A. Schwadron, and R. M. Skoug (2008), Weaker solar wind from the polar coronal holes and the whole sun, Geophys. Res. Lett., 35, L18103, doi:10.1029/2008GL034896.

Schwenn, R. (1990), Large-scale structure of the interplanetary medium, in Physics of the Inner Heliosphere, vol. 1, Large-Scale Phenomena, edited by R. Schwenn and E. Marsch, chap. 3, pp. 99-181, Springer, Berlin. von Steiger, R. (2008), The solar wind throughout the solar cycle, in The Heliosphere Through the Solar Activity Cycle, edited by A. Balogh, L. J. Lanzerotti, and S. T. Suess, chap. 3, pp. 41-78, doi:10.1007/9783-540-74302-63, Springer Praxis, Chichester, U. K.

von Steiger, R., and J. Geiss (1989), Supply of fractionated gases to the corona, Astron. Astrophys., 225, 222-238.

von Steiger, R., and T. H. Zurbuchen (2010), Polar coronal holes during the past solar cycle: Ulysses observations, J. Geophys. Res., doi:10.1029/ 2010JA015835, in press.

von Steiger, R., N. A. Schwadron, L. A. Fisk, J. Geiss, G. Gloeckler, S. Hefti, B. Wilken, R. F. Wimmer-Schweingruber, and T. H. Zurbuchen (2000), Composition of quasi-stationary solar wind flows from Ulysses/ Solar Wind Ion Composition Spectrometer, J. Geophys. Res., 105, 27,217-27,238, doi:10.1029/1999JA000358.
Wenzel, K.-P., R. G. Marsden, D. E. Page, and E. J. Smith (1992), The Ulysses mission, Astron. Astrophys. Suppl. Ser., 92, 207-219.

Zurbuchen, T. H. (2007), A new view of the coupling of the Sun and the heliosphere, Annu. Rev. Astron. Astrophys., 45, 297, doi:10.1146/ annurev.astro.45.010807.154030.

Zurbuchen, T. H., L. A. Fisk, G. Gloeckler, and R. von Steiger (2002), The solar wind composition throughout the solar cycle: A continuum of dynamic states, Geophys. Res. Lett., 29(9), 1352, doi:10.1029/ 2001 GL013946.

D. J. McComas, Space Science and Engineering Division, Southwest Research Institute, PO Drawer 28510, San Antonio, TX 78228, USA. (dmccomas@swri.edu)

R. von Steiger, International Space Science Institute, Hallerstrasse 6, CH-3012 Bern, Switzerland. (vsteiger@issibern.ch)

T. H. Zurbuchen, Department of Atmospheric, Oceanic and Space Sciences, University of Michigan, 2455 Hayward St., Ann Arbor, MI 48109, USA. (thomasz@umich.edu) 